\title{
Sodium-Glucose Linked Transporter Inhibitors as a Cause of Euglycemic Diabetic Ketoacidosis on a Background of Starvation
}

\author{
Abubakar Tauseef ${ }^{1}$, Muhammad Sohaib Asghar ${ }^{2}$, Maryam Zafar $^{3}$, Noman Lateef ${ }^{1}$, Joseph \\ Thirumalareddy ${ }^{4}$ \\ 1. Internal Medicine, Creighton University, Omaha, USA 2. Internal Medicine, Dow International Medical College, Dow \\ University Hospital, Dow University of Health Sciences, Karachi, PAK 3. Medicine, Dow International Medical College, \\ Dow University Hospital, Dow University of Health Sciences, Karachi, PAK 4. Hospital Medicine, Creighton University, \\ Omaha, USA
}

Corresponding author: Abubakar Tauseef, abubakartauseef93@gmail.com

\begin{abstract}
We present a case of a male diabetic patient with one of the most well-known major complications of diabetes mellitus (DM), i.e., diabetic ketoacidosis (DKA). The finding of euglycemic DKA, or DKA with blood glucose levels of less than $200 \mathrm{mg} / \mathrm{dL}$, is a rare occurrence especially in patients with type II DM. He presented with hypotension and increased anion gap metabolic acidosis on a background of keto diet for weight loss and recent use of sodium-glucose linked transporter inhibitors. He was successfully managed with dextrose water, insulin infusion, and potassium replacement. A ketogenic diet consists of high fat, low carbohydrate, and adequate protein regimen that sends the body into a state of starvation in which high glucagon and low insulin levels lead to the activation of other counter-regulatory hormones, such as epinephrine and cortisol, that causes a rise in the level of free fatty acids in the blood increasing ketone body production. Rarely, sodium-glucose linked transport inhibitors can also cause DKA, with euglycemia instead of hyperglycemia. The finding of plasma glucose levels within normal range prompted patients to maintain and sometimes even lower their insulin dose; even their providers were often misled by the euglycemia that resulted in delayed diagnosis and treatment. Thus, it is imperative to stay aware of the possible clinical presentations in order to intervene in a timely manner and prevent further progression and complications.
\end{abstract}

Received 08/19/2020

Review began 08/22/2020 Review ended 08/26/2020 Published 08/27/2020

(c) Copyright 2020

Tauseef et al. This is an open access article distributed under the terms of the Creative Commons Attribution License CC-BY 4.0., which permits unrestricted use, distribution, and reproduction in any medium, provided the original author and source are credited.
Categories: Endocrinology/Diabetes/Metabolism, Emergency Medicine, Internal Medicine Keywords: diabetes mellitus, euglycemic, sglt-2, diabetic ketoacidosis, glucose, insulin

\section{Introduction}

One of the most well-known major complications of diabetes mellitus (DM) is the development of diabetic ketoacidosis (DKA). DKA most commonly presents in individuals with type I DM with raised plasma glucose levels in the characteristic triad of hyperglycemia, ketosis, and metabolic acidosis [1]. The finding of euglycemic DKA, or DKA with blood glucose levels of less than $200 \mathrm{mg} / \mathrm{dL}$, is a rare occurrence especially in patients with type II DM [2-4]. In our case, the patient was a type II diabetic who followed a ketogenic diet for three weeks and lost $17 \mathrm{lbs}$. A diagnosis of type I DM was ruled out, and the patient was found to be in euglycemic ketoacidosis likely triggered by his dietary habits in combination with the use of empagliflozin.

A ketogenic diet consists of high fat, low carbohydrate, and adequate protein regimen that sends the body into a state of starvation in which high glucagon and low insulin levels lead to the activation of other counter-regulatory hormones, such as epinephrine and cortisol, that causes a rise in the level of free fatty acids in the blood increasing ketone body production [5,6]. The risk for the development of metabolic ketoacidosis increases as glycogen is depleted to maintain normal plasma glucose levels and ketones become the predominant means of metabolism in the body [7].

Sodium-glucose linked transport inhibitors work by preventing the absorption of glucose through the nephrons, hence enhancing the excretion of excess glucose through the urine [8]. It has been approved for usage in treating DM since 2013. Recently, it has also been approved to treat heart failure in type II DM patients with a reduction in cardiovascular complications and death [9].

\section{Case Presentation}

A 61-year-old male with a significant past medical history of type II DM, being managed by metformin and insulin for the last 10 years, prescribed empagliflozin two months back, presented to the emergency department with hypotension. He mentioned that he started a keto diet three weeks back and lost $17 \mathrm{lbs}$. He denied polyuria, polydipsia, polyphagia, headache, nausea, vomiting, headache, altered level, and loss of consciousness. His physical examination was unremarkable. He was started on fluid replacement with Ringer lactate, and workup for blood sugars, complete blood count (CBC) with differential, basal metabolic 
profile (BMP), lactic acid, arterial blood gases, beta-hydroxybutyrate, serum osmolarity, and procalcitonin were sent.

Laboratory investigation showed increased anion gap metabolic acidosis with a pH of 7.11 (normal: 7.357.45), serum bicarbonate $\left(\mathrm{HCO}_{3}\right)$ of $7 \mathrm{mEq} / \mathrm{L}$ (normal: $20-28$ ) and an anion gap of $20 \mathrm{mEq} / \mathrm{L}$ (normal: 8-16), his lactate level came out to be $6.1 \mathrm{mg} / \mathrm{dL}$ (4.5-19.8), blood sugars were $90 \mathrm{mg} / \mathrm{dL}$ (normal: 80-120), serum osmolarity of $310 \mathrm{mOsm} / \mathrm{kg} \mathrm{H}_{2} \mathrm{O}$ (normal: $285-295$ ), and beta-hydroxybutyrate of $>4.5 \mathrm{mmol} / \mathrm{L}$ (normal: 0.40.5 ). The rest of the laboratory investigations were unremarkable. He got diagnosed with DKA but with completely normal serum glucose levels, it could be secondary to his keto diet/starvation or due to the use of empagliflozin. He got transferred to the Intensive Care Unit (ICU) for further management and started on 5\% dextrose water (D5W)/0.45\% normal saline with $20 \mathrm{mEq}$ of potassium fluids with insulin infusion at a rate of $1 \mathrm{unit} / \mathrm{kg} / \mathrm{hr}$, and metformin and empagliflozin were discontinued. His blood sugars were monitored every two hourly which mostly stayed between 90 and $100 \mathrm{mg} / \mathrm{dL}$. Therefore, his urine was sent for ketones which returned positive. The chest X-ray was unremarkable. The endocrinology department was consulted, and they recommended continuing the same management plan with workup for ruling out type I DM, which was negative. His management was continued for 24 hours with a very little improvement, as his $\mathrm{pH}$ increased to 7.20 with an anion gap of $15 \mathrm{mEq} / \mathrm{L}, \mathrm{HCO}_{3}$ of $11 \mathrm{mEq} / \mathrm{L}$, lactic acid of $4.7 \mathrm{mg} / \mathrm{dL}$, blood sugars of $95 \mathrm{mg} / \mathrm{dL}$, and serum osmolarity of $305 \mathrm{mOsm} / \mathrm{kg} \mathrm{H}_{2} \mathrm{O}$. He was continued on the same management plan for another 24 hours but with an increased rate of fluid to $200 \mathrm{~mL} / \mathrm{hr}$. Anion gap was closed at $8 \mathrm{mEq} / \mathrm{L}$, with a pH of 7.37, $\mathrm{HCO}_{3}$ of $20 \mathrm{mEq} / \mathrm{L}$, lactate came down to $0.7 \mathrm{mg} / \mathrm{dL}$, blood sugars stayed between 90 and $100 \mathrm{mg} / \mathrm{dL}$, and serum osmolarity decreased to $295 \mathrm{mOsm} / \mathrm{kg} \mathrm{H} \mathrm{H}_{2} \mathrm{O}$.

The patient was started on subcutaneous insulin detemir with an overlap of an insulin infusion for two hours and infusion was weaned off afterward. A dietician was consulted, who discontinued his keto diet and started him on a regular diet. The patient was discharged home with subcutaneous insulin detemir daily and metformin 1,000 mg BID with a follow-up in one week with the critical care team and endocrinologist. His follow-up was unremarkable with blood sugar levels of $110 \mathrm{mg} / \mathrm{dL}$ in the last one week and no acidosis. The patient was counseled regarding all the options for reducing his weight which was his concern and was educated about how to implement keto diet in his life in order to prevent any further episodes of severe acidosis.

\section{Discussion}

Sodium-glucose linked transport inhibitors, such as empagliflozin, are approved for clinical use in the treatment of type II DM in adults; they induce an increase in the excretion of glucose by the kidneys resulting in lower blood glucose levels [8]. Some adverse effects associated with these inhibitors include dehydration, increased cholesterol levels, yeast infections, and kidney problems. Rarely, they can also cause DKA, and interestingly, many of those cases revealed euglycemia instead of hyperglycemia [9].

A few studies report euglycemic DKA due to a low-carbohydrate diet, including one conducted in Japan in which the patient was on canagliflozin and after severely restricting her carbohydrate intake for six days, presented with progressive dyspnea and altered mental status; laboratory investigations revealed severe ketoacidosis and euglycemia [10]. Another case in Taiwan involved a type II diabetic patient on dapagliflozin with a history of poor oral intake for one week attributed to severe toothache who came with the complaints of weakness, dyspnea, nausea, vomiting, and mild abdominal pain; severe metabolic acidosis with an elevated anion gap was discovered upon further investigation [11].

Other causes of euglycemic DKA include pregnancy, cocaine abuse, pancreatitis, cirrhosis, and sepsis; thus, normal plasma glucose levels should not be sufficient to rule out the possibility of DKA in a patient especially if he/she is on sodium-glucose linked transport inhibitors [12,13]. Although the risk of euglycemic DKA in type II DM associated with sodium-glucose linked transport inhibitors is low enough to have an "acceptable frequency" according to the American Diabetes Association, it may be higher in patients with long-standing type II DM during periods of stress such as post-surgery and prolonged starvation [14]. The first case series of euglycemic DKA in the United States found that the common feature in the nine cases presented was that despite the development of ketoacidosis, the finding of plasma glucose levels within normal range prompted patients to maintain and sometimes even lower their insulin dose; even their providers were often misled by the euglycemia that resulted in delayed diagnosis and treatment [15]. Thus, it is imperative to stay aware of the possible clinical presentations in order to intervene in a timely manner and prevent further progression and complications.

\section{Conclusions}

Our case presented with hypotension and increased anion gap metabolic acidosis on a background of controlled type II diabetes with the use of sodium-glucose linked transport inhibitors and keto diet. With blood sugar levels within normal limits, he was managed as a case of euglycemic ketoacidosis, which is a rare side effect of sodium-glucose linked transport inhibitors as well as reported in paucity with starvation in known diabetics. He was successfully managed with D5W/0.45\% normal saline, insulin infusion, and potassium replacement. 


\section{Additional Information \\ Disclosures}

Human subjects: Consent was obtained by all participants in this study. Conflicts of interest: In compliance with the ICMJE uniform disclosure form, all authors declare the following: Payment/services info: All authors have declared that no financial support was received from any organization for the submitted work. Financial relationships: All authors have declared that they have no financial relationships at present or within the previous three years with any organizations that might have an interest in the submitted work. Other relationships: All authors have declared that there are no other relationships or activities that could appear to have influenced the submitted work.

\section{References}

1. Tran TT, Pease A, Wood AJ, Zajac JD, Mårtensson J, Bellomo R, Ekinci EI: Review of evidence for adult diabetic ketoacidosis management protocols. Front Endocrinol. 2017, 8:106. 10.3389/fendo.2017.00106

2. Ogawa W, Sakaguchi K: Euglycemic diabetic ketoacidosis induced by SGLT2 inhibitors: possible mechanism and contributing factors. J Diabetes Investig. 2016, 7:135-138. 10.1111/jdi.12401

3. Modi A, Agrawal A, Morgan F: Euglycemic diabetic ketoacidosis: a review . Curr Diabetes Rev. 2017, 13:315321. 10.2174/1573399812666160421121307

4. Gammons DT, Counselman FL: Sodium-glucose cotransporter-2 induced diabetic ketoacidosis with minimal hyperglycemia. Clin Pract Cases Emerg Med. 2018, 2:47-50. 10.5811/cpcem.2017.12.36213

5. Svart MV, Voss TS, Bayat M, Madsen LR, Andersen LT, Poulsen PL, Møller N: Rare presentations of ketoacidosis: diabetic ketoalkalosis and ketoacidosis secondary to fasting and muscular dystrophy. Clin Diabetes. 2015, 33:37-39. 10.2337/diaclin.33.1.37

6. Blanco JC, Khatri A, Kifayat A, Cho R, Aronow WS: Starvation ketoacidosis due to the ketogenic diet and prolonged fasting: a possibly dangerous diet trend. Am J Case Rep. 2019, 20:1728-1731. 10.12659/AJCR.917226

7. Joseph F, Anderson L, Goenka N, Vora J: Starvation-induced true diabetic euglycemic ketoacidosis in severe depression. J Gen Intern Med. 2009, 24:129-131. 10.1007/s11606-008-0829-0

8. Levine MJ: Empagliflozin for type 2 diabetes mellitus: an overview of phase 3 clinical trials . Curr Diabetes Rev. 2017, 13:405-423. 10.2174/1573399812666160613113556

9. Rashid O, Farooq S, Kiran Z, Islam N: Euglycaemic diabetic ketoacidosis in a patient with type 2 diabetes started on empagliflozin. BMJ Case Rep. 2016, 2016:bcr2016215340. 10.1136/bcr-2016-215340

10. Fukuyama Y, Numata K, Yoshino K, Santanda, T, Funakoshi H: Euglycemic diabetic ketoacidosis due to a strict low-carbohydrate diet during treatment with sodium-glucose cotransporter 2 inhibitors. Acute Med Surg. 2020, $7:$ :e480. 10.1002/ams2.480

11. Chou YM, Seak CJ, Goh ZNL, Seak JCY, Seak CK, Lin CC: Euglycemic diabetic ketoacidosis caused by dapagliflozin: a case report. Medicine. 2018, 97:e11056. 10.1097/MD.0000000000011056

12. Lucero P, Chapela S: Euglycemic diabetic ketoacidosis in the ICU: 3 case reports and review of literature . Case Rep Crit Care. 2018, 2018:1747850. 10.1155/2018/1747850

13. Rawla, P, Vellipuram AR, Bandaru SS, Pradeep Raj J: Euglycemic diabetic ketoacidosis: a diagnostic and therapeutic dilemma. Endocrinol Diabetes Metab Case Rep. 2017, 2017:17-0081. 10.1530/EDM-17-0081

14. Rosenstock J, Ferrannini E: Euglycemic diabetic ketoacidosis: a predictable, detectable, and preventable safety concern with SGLT2 inhibitors. Diabetes Care. 2015, 38:1638-1642. 10.2337/dc15-1380

15. Peters AL, Buschur EO, Buse JB, Cohan P, Diner JC, Hirsch IB: Euglycemic diabetic ketoacidosis: a potential complication of treatment with sodium-glucose cotransporter 2 inhibition. Diabetes Care. 2015, 38:16871693. $10.2337 /$ dc15-0843 